This item was submitted to Loughborough's Research Repository by the author.

Items in Figshare are protected by copyright, with all rights reserved, unless otherwise indicated.

\title{
Eckardt loci on hypersurfaces
}

PLEASE CITE THE PUBLISHED VERSION

http://dx.doi.org/10.1080/00927872.2014.910798

\section{PUBLISHER}

Taylor \& Francis

\section{VERSION}

AM (Accepted Manuscript)

\section{PUBLISHER STATEMENT}

This work is made available according to the conditions of the Creative Commons Attribution-NonCommercialNoDerivatives 4.0 International (CC BY-NC-ND 4.0) licence. Full details of this licence are available at: https://creativecommons.org/licenses/by-nc-nd/4.0/

\section{LICENCE}

CC BY-NC-ND 4.0

\section{REPOSITORY RECORD}

Coskun, Izzet, and Artie Prendergast-Smith. 2019. "Eckardt Loci on Hypersurfaces". figshare. https://hdl.handle.net/2134/17284. 


\title{
ECKARDT LOCI ON HYPERSURFACES
}

\author{
IZZET COSKUN AND ARTIE PRENDERGAST-SMITH
}

\begin{abstract}
We compute the dimensions and cohomology classes of the loci on a general hypersurface where the second fundamental form has rank at most $r$. We also determine the number of hypersurfaces in a general pencil in $\mathbb{P}^{n}$, with $n=\left(\begin{array}{c}q+1 \\ 2\end{array}\right)$,

that contain a point where the second fundamental form has rank $n-1-q$. These results generalize many classical formulae.
\end{abstract}

\section{Contents}

1. Introduction

2. Basic properties of Eckardt loci

3. Eckardt loci on general hypersurfaces

4. Eckardt loci in a general pencil of hypersurfaces

References

\section{INTRODUCTION}

Let $X \subset \mathbb{P}^{n}$ be a smooth projective variety of codimension $m$ over the complex numbers $\mathbb{C}$. Let $G: X \rightarrow \mathbb{G}(n-m, n)$ denote the Gauss map, which associates to a point $x \in X$ the projective tangent space of $X$ at $x$. The differential of $G$ at $x$ is given by the second fundamental form $\left|I I_{x}\right|$, which is an $(m-1)$-dimensional linear system of quadrics in $\mathbb{P} T_{x} X$ (see $[\mathrm{GH}]$ ). The second fundamental form plays a central role in studying the projective geometry of $X$. For example, the degeneracy of tangential, secant and dual varieties (see [GH]) and the properties of linear spaces on $X$ (see [CS], [LR]) are very closely tied to the second fundamental form.

Definition 1.1. Define the rank $r(x)$ of the second fundamental form at $x \in X$ to be the maximal rank of a quadric in the linear system $\left|I I_{x}\right|$.

Landsberg [L, Corollary 4.2] proved that if the singular locus of $X \subset \mathbb{P}^{n}$ has dimension $b$, then, at a general point $x \in X$, the dimension of the singular locus of a general quadric in $\left|I I_{x}\right|$ is bounded above by $m+b$. Moreover, at a general point $x$ of a smooth complete intersection, $r(x)=n-m[\mathrm{~L}$, Theorem 6.1]. In this paper, we study the dependence of $r(x)$ on $x \in X$.

In the classical literature, points on a cubic surface where three lines meet are called Eckardt points. These are precisely the points of a cubic surface where the second fundamental form vanishes. Inspired by the classical terminology, we make the following definition.

During the preparation of this article the first author was partially supported by the NSF CAREER grant DMS-0950951535, and an Alfred P. Sloan Foundation Fellowship. 
Definition 1.2. An $r$-Eckardt point is a point $x \in X$ such that $r(x) \leq r$. We denote the locus of $r$-Eckardt points on $X$ by $X_{r}$.

In this paper, we compute the dimension and cohomology class of $X_{r}$ when $X$ is a general hypersurface. We also compute the number of $r$-Eckardt points in a general pencil of hypersurfaces when the expected number is finite. We now summarize our results.

Theorem 1.3. Let $X \subset \mathbb{P}^{n}$ be a general hypersurface of degree $d>2$. Then

$$
\operatorname{dim}\left(X_{r}\right)=\max \left(-1, n-1-\left(\begin{array}{c}
n-r \\
2
\end{array}\right)\right) .
$$

In particular, if $\left(\begin{array}{c}n-r \\ 2\end{array}\right) \geq n$, then $X_{r}=\varnothing$. Furthermore, if $\left(\begin{array}{c}n-r \\ 2\end{array}\right) \geq n$, then the locus of smooth hypersurfaces in the Hilbert scheme $\mathbb{P}^{\left(\begin{array}{c}n+d \\ d\end{array}\right)-1}$ that have an $r$-Eckardt point is irreducible and has codimension $\left(\begin{array}{c}n-r \\ 2\end{array}\right)-n+1$.

More generally, in \$2, we bound the dimensions of Eckardt loci in complete intersections. Unfortunately, as the codimension of the complete intersection increases our results become less sharp.

The locus of cubic surfaces that have an Eckardt point (a 0-Eckardt point in our terminology) plays an essential role in studying the geometry of the moduli space of cubic surfaces (see [Ros]). In analogy, we expect loci of hypersurfaces with $r$-Eckardt points to play an important role in the geometry and cohomology of the moduli spaces of higher-degree hypersurfaces. In the last section, we discuss the problem of computing the classes of the loci of hypersurfaces that have an $r$-Eckardt point. In Theorem 3.1. we show that on a general hypersurface $X \subset \mathbb{P}^{n}$ of degree $d \geq 2$ the locus $X_{n-1-q}$ has degree

$$
\left(\prod_{i=0}^{q-1} \frac{\left(\begin{array}{c}
n+1+i \\
q-i
\end{array}\right)}{\left(\begin{array}{c}
2 i+1 \\
i
\end{array}\right)}\right) d(d-2)^{\frac{q(q+1)}{2}} .
$$

Finally, in Theorem 4.1, we show that the number of hypersurfaces that have an $(n-1-q)$-Eckardt point in a general pencil of hypersurfaces of degree $d \geq 2$ in $\left.\mathbb{P}^{(q+1} 2\right)$ is

$$
\left(\prod_{i=0}^{q-1} \frac{\left(\begin{array}{c}
n+1+i \\
q-i
\end{array}\right)}{\left(\begin{array}{c}
2 i+1 \\
i
\end{array}\right)}\right)(d-2)^{n-1}((n+1) d-2) .
$$

The main new feature of this computation is the need to extend the symmetric Porteous formula of Harris-Tu [HT] to the context of coherent sheaves that occur in our set up.

Acknowledgements: We are grateful to J.M. Landsberg, D. Lehavi, Z. Wolscke, and D. Zeilberger for helpful correspondences. Special thanks to Christoph Koutschan, who provided the algorithmic expertise necessary to prove Lemma 4.3 .

\section{BASIC PROPERTIES OF ECKARDT LOCI}

In this section, we discuss basic geometric properties of the $r$-Eckardt loci on hypersurfaces and, more generally, on complete intersections. We refer the reader to [GH2, Chapter 6] and [C] for details concerning the geometry of quadrics. 
Notation 2.1. Let $N=\left(\begin{array}{c}n+d \\ d\end{array}\right)-1$. Then the Hilbert scheme parameterizing degree $d$ hypersurfaces in $\mathbb{P}^{n}$ is isomorphic to $\mathbb{P}^{N}$. Let $\mathcal{X}$ denote the universal hypersurface of degree $d$ in $\mathbb{P}^{n}$ parameterizing pairs $\left\{(p, X) \mid p \in X \subset \mathbb{P}^{n}\right\}$, where $X$ is a hypersurface of degree $d$ and $p$ is a point of $X$. The universal hypersurface $\mathcal{X}$ admits two natural projections $\pi_{1}$ and $\pi_{2}$ to $\mathbb{P}^{n}$ and $\mathbb{P}^{N}$, respectively. Let $\mathcal{U}$ be the open subset in $\mathcal{X}$ where $X$ is smooth. Observe that $\mathcal{U}$ is the inverse image under $\pi_{2}$ of the complement of the discriminant in $\mathbb{P}^{N}$.

In order to study $r$-Eckardt loci, we linearize the problem. Let $I$ be the incidence correspondence

$$
\left\{(p, X, T, Q) \mid(p, X) \in \mathcal{U}, T=T_{p} X, I I_{p}=Q \subset \mathbb{P} T\right\}
$$

where $X$ is a smooth hypersurface, $p$ is a point of $X, T$ is the tangent space to $X$ at $p$ and $Q$ is the second fundamental form of $X$ at $p$. The incidence correspondence $I$ has natural subsets $I_{r}$ defined by requiring that the rank of $Q$ be equal to $r$.

Proof of Theorem 1.3. In order to prove the theorem, we consider the projection $\pi_{1,3,4}$ defined on $I_{r}$ forgetting the second factor. The locus of quadrics of corank $q$ in $\mathbb{P}^{n-1}$ is irreducible and has codimension $\left(\begin{array}{c}q+1 \\ 2\end{array}\right)$. Hence, the space

$$
A=\{(p, T, Q) \mid p \in T, Q \subset \mathbb{P} T, \operatorname{rank}(Q)=r\}
$$

is irreducible and has dimension

$$
2 n-1+\left(\begin{array}{l}
n \\
2
\end{array}\right)-1-\left(\begin{array}{c}
n-r \\
2
\end{array}\right) .
$$

The fibers of $\pi_{1,3,4}$ are open subspaces of linear spaces. By the homogeneity of $A$, we may assume that $p=\left[\begin{array}{l}1: 0: \cdots: 0] \\ 0\end{array}\right]$ and $T$ is defined by $x_{n}=0$. We can take $Q$ to be defined by the equation $x_{1}^{2}+x_{2}^{2}+\cdots+x_{r}^{2}=0$. Then the Taylor expansion of any hypersurface (in the affine coordinates obtained by setting $x_{0}=1$ ) has the form

$$
c_{1} x_{n}+c_{2}\left(x_{1}^{2}+x_{2}^{2}+\cdots+x_{r}^{2}\right)+x_{n} L\left(x_{1}, \cdots, x_{n}\right)+\text { h. o. t. },
$$

where $c_{1}$ and $c_{2}$ are constants and $L$ is a linear form. We conclude that the fibers of $\pi_{1,3,4}$ are irreducible of dimension

$$
\left(\begin{array}{c}
n+d \\
d
\end{array}\right)-1-n-\left(\begin{array}{l}
n \\
2
\end{array}\right)+1
$$

By the theorem on the dimension of fibers, we conclude that $I_{r}$ is irreducible and

$$
\operatorname{dim}\left(I_{r}\right)=\left(\begin{array}{c}
n+d \\
d
\end{array}\right)-1+n-1-\left(\begin{array}{c}
n-r \\
2
\end{array}\right)
$$

Most of Theorem 1.3 follows from this calculation. First, observe that since $I_{r}$ is irreducible, the image of the second projection $\pi_{2}: I_{r} \rightarrow \mathbb{P}^{N}$ is also irreducible. We conclude that the locus in the Hilbert scheme of smooth hypersurfaces that have an $r$-Eckardt point is irreducible.

If $\left(\begin{array}{c}n-r \\ 2\end{array}\right)>n-1$, then $\operatorname{dim}\left(I_{r}\right)<N$. Hence, the projection to the Hilbert scheme $\mathbb{P}^{N}$ cannot be dominant. We conclude that a general hypersurface does not contain an $r$-Eckardt point if $\left(\begin{array}{c}n-r \\ 2\end{array}\right)>n-1$. Now we do a more delicate analysis to prove that when $\left(\begin{array}{c}n-r \\ 2\end{array}\right)>n-1$ the second projection maps $I_{r}$ birationally onto its image. In particular, among smooth hypersurfaces that have an $r$-Eckardt point, the general one has a unique $r$-Eckardt point. 
Let $J_{r}$ be the incidence correspondence

$$
\left\{\left(p_{1}, p_{2}, X, T_{1}, T_{2}, Q_{1}, Q_{2}\right) \mid\left(p_{i}, X\right) \in \mathcal{U}, p_{1} \neq p_{2}, T_{p_{i}} X=T_{i}, I I_{p_{i}}=Q_{i} \subset \mathbb{P} T_{i}, \operatorname{rank}\left(Q_{i}\right)=r\right\} .
$$

Let $\pi_{\hat{3}}$ be the projection that forgets the third factor and let $\pi_{\hat{3}, \hat{\sigma}, \hat{7}}$ be the projection that forgets the third, sixth and seventh factors. The image of $\pi_{\hat{3}, \hat{b}, \hat{\gamma}}$ is no longer homogeneous, but decomposes into the following $\mathbb{P} G L(n+1)$-orbits:

(1) $p_{i} \notin T_{j}$, for $i \neq j$

(2) $p_{1} \in T_{2}$, but $p_{2} \notin T_{1}$ (and the similar locus obtained by interchanging the indices 1 and 2),

(3) $p_{i} \in T_{j}$, but $T_{1} \neq T_{2}$,

(4) $T_{1}=T_{2}$.

We need to show that the potential components of $J_{r}$ lying over each of these loci have dimensions smaller than the dimension of $I_{r}$. The calculations are almost identical for each of these loci, so we will carry them out for the locus (1) in detail and briefly indicate the changes necessary in the other cases. The incidence correspondence $\left\{\left(p_{1}, p_{2}, T_{1}, T_{2}\right) \mid p_{i} \in T_{i}\right\}$ has dimension $4 n-2$. The loci (2), (3), and (4) have codimension 1,2 , and $n$, respectively, in this incidence correspondence. The choice of the two quadrics $Q_{i} \subset \mathbb{P} T_{i}$, for $i=1,2$, adds

$$
2\left(\begin{array}{l}
n \\
2
\end{array}\right)-2-2\left(\begin{array}{c}
n-r \\
2
\end{array}\right)
$$

to the dimension. Hence, we determine the dimension of the image of the projection $\pi_{\hat{3}}$ to be

$$
4 n-2+2\left(\begin{array}{l}
n \\
2
\end{array}\right)-2-2\left(\begin{array}{c}
n-r \\
2
\end{array}\right)
$$

Taking $p_{1}=[1: 0: \cdots: 0]$ and $T_{1}=\left\{x_{n}=0\right\}$ and $p_{2}=[0: 0: \cdots: 0: 1]$ and $T_{2}=\left\{x_{0}=0\right\}$ and specifying two quadrics of rank $r$ in $\mathbb{P} T_{i}$, we see that the fiber of $\pi_{\hat{3}}$ is an open subset in a linear space of dimension

$$
\left(\begin{array}{c}
n+d \\
d
\end{array}\right)-1-2 n-2\left(\begin{array}{l}
n \\
2
\end{array}\right)+2
$$

Similar calculations for the loci (2), (3) and (4) show that the dimension of the fibers of $\pi_{\hat{3}}$ remain bounded by the same quantity. Therefore, if $\left(\begin{array}{c}n-r \\ 2\end{array}\right)>n-1$, then $\operatorname{dim}\left(J_{r}\right)<\operatorname{dim}\left(I_{r}\right)$. Hence, the projection from $J_{r}$ to $I_{r}$ cannot be dominant. Consequently, when $\left(\begin{array}{c}n-r \\ 2\end{array}\right)>n-1$, a general hypersurface that has an $r$-Eckardt point has only one $r$-Eckardt point.

Now assume that $\left(\begin{array}{c}n-r \\ 2\end{array}\right) \leq n-1$. If the projection $\pi_{2}: I_{r} \rightarrow \mathbb{P}^{N}$ is dominant, then the theorem on the dimension of fibers implies that $\operatorname{dim}\left(X_{r}\right)=n-1-\left(\begin{array}{c}n-r \\ 2\end{array}\right)$ for a general $X$. In Theorem 3.1, we will compute the class of the degeneracy locus of the second fundamental form. Since this class is non-zero when $d>2$, we conclude that $\pi_{2}$ must be dominant. This concludes the proof of the theorem.

We now generalize these results to higher codimension complete intersections. Since we have already discussed the case of hypersurfaces, for the rest of the section we assume that the codimension $m \geq 2$.

Notation 2.2. Given positive integers $\mu, \nu$ and $r$, let $D(\mu, \nu, r)$ be the locus in $\mathbb{G}\left(\mu,\left(\begin{array}{c}\nu+2 \\ 2\end{array}\right)-1\right)$ parameterizing $\mu$-dimensional linear systems of quadrics in $\mathbb{P}^{\nu}$ such that every member has rank bounded above by $r$. Let $\delta(\mu, \nu, r)$ denote the dimension of the locus $D(\mu, \nu, r)$.

The following is a generalization of Theorem 1.3 to the case of complete intersections. 
Proposition 2.3. Let $X$ be a general smooth complete intersection of codimension $m$ and type $\left(d_{1}, \ldots, d_{m}\right)$, where $2<d_{1} \leq d_{2} \leq \cdots \leq d_{m}$, in $\mathbb{P}^{n}$. Then

$$
\operatorname{dim}\left(X_{r}\right) \leq \max \left(-1, n+\delta(m-1, n-m-1, r)-m\left(\left(\begin{array}{c}
n-m+1 \\
2
\end{array}\right)-m+1\right)\right) .
$$

In particular, $X$ has no $r$-Eckardt points if

$$
n-m<m\left(\left(\begin{array}{c}
n-m+1 \\
2
\end{array}\right)-m\right)-\delta(m-1, n-m-1, r) .
$$

Proof. Let $\mathcal{H}$ be the locus in the Hilbert scheme parameterizing smooth complete intersections. Note that $\mathcal{H}$ can be realized as an open subset of a tower of Grassmannian bundles. Let $\mathcal{U}$ be the universal smooth complete intersection parameterizing pairs $(p, X)$, where $X$ is a smooth complete intersection and $p$ is a point of $X$. Let $I$ be the incidence correspondence

$$
I:=\left\{(p, X, T, W) \mid(p, X) \in \mathcal{U}, T=T_{p} X, I I_{p}=W\right\} .
$$

Let $I_{r}$ be the subvariety of $I$ defined by requiring every member of $W$ to have rank at most $r$. Let $\pi$ be the projection that forgets the second factor. Then the dimension of the image of $\pi$ is $n+(n-m) m+$ $\delta(m-1, n-m-1, r)$. On the other hand, the fiber dimension of the projection is

$$
\operatorname{dim}(\mathcal{H})-n-(n-m)(m-1)-m\left(\left(\begin{array}{c}
n-m+1 \\
2
\end{array}\right)-m\right)
$$

We conclude that $\operatorname{dim}\left(I_{r}\right)=\operatorname{dim}(\mathcal{H})+n-m+\delta(m-1, n-m-1, r)-m\left(\left(\begin{array}{c}n-m+1 \\ 2\end{array}\right)-m\right)$. If $\operatorname{dim}\left(I_{r}\right)<$ $\operatorname{dim}(\mathcal{H})$, then the projection of $I_{r}$ to $\mathcal{H}$ cannot be dominant. Hence, a general complete intersection has no $r$-Eckardt points if $n-m<m\left(\left(\begin{array}{c}n-m+1 \\ 2\end{array}\right)-m\right)-\delta(m-1, n-m-1, r)$. Similarly, as long as the second projection is dominant, by the theorem on the dimension of fibers, the dimension of $X_{r}$ is $n-m+\delta(m-1, n-m-1, r)-m\left(\left(\begin{array}{c}n-m+1 \\ 2\end{array}\right)-m\right)$.

In view of Proposition 2.3, it is interesting to calculate $\delta(\mu, \nu, r)$. The literature on understanding linear systems of quadrics with fixed rank is extensive (e.g., [IL], [M], [W1] and [W2]). However, the main focus seems to be on finding the largest dimensional linear system where all the quadrics have a fixed rank.

We now give some bounds on $\delta(\mu, \nu, r)$. Since we do not know sharp bounds, we will opt for simplicity over optimizing our techniques. We can obtain an easy lower bound on $\delta(\mu, \nu, r)$ by considering linear systems of quadrics that have a fixed $(\nu-r)$-dimensional singular locus. Assume that $\mu \leq\left(\begin{array}{c}r+1 \\ 2\end{array}\right)-1$. In $\mathbb{P}^{\nu}$, fix a linear subspace $L_{1}$ of dimension $\nu-r$ and a disjoint linear subspace $L_{2}$ of dimension $r-1$. Given a $\mu$-dimensional linear system of quadrics in $L_{2}$, taking the cone over each of these quadrics with vertex $L_{1}$, we obtain a $\mu$-dimensional linear system of quadrics in $\mathbb{P}^{\nu}$ where each member has rank at most $r$. Since we can vary $L_{1}$ in an open subset of the Grassmannian $\mathbb{G}(\nu-r, \nu)$, we see that

$$
(\mu+1)\left(\left(\begin{array}{c}
r+1 \\
2
\end{array}\right)-\mu-1\right)+r(\nu-r+1) \leq \delta(\mu, \nu \cdot r) .
$$

Remark 2.4. The locus of rank one quadrics is parameterized by the quadratic Veronese embedding $V_{2, \nu}$ of $\mathbb{P}^{\nu}$ in $\mathbb{P}^{\left(\begin{array}{c}\nu+2 \\ 2\end{array}\right)-1}$. More generally, the locus of rank $r$ quadrics is parameterized by the $r$-secant variety 
$R(r, \nu)$ to $V_{2, \nu}$. The variety $R(r, \nu)$ is swept-out by linear spaces $\mathbb{P}^{\left(\begin{array}{c}r+1 \\ 2\end{array}\right)-1}$. The lower bound on $\delta(\mu, \nu, r)$ is obtained by taking $\mu$-dimensional linear spaces contained in one of the $\mathbb{P}^{\left(\begin{array}{c}r+1 \\ 2\end{array}\right)-1}$.

Now we find some upper bounds for $\delta(\mu, \nu, r)$. Let $\Lambda$ be a $\mu$-dimensional linear system of quadrics in $\mathbb{P}^{\nu}$ such that every member has rank at most $r$. Then $\Lambda$ is contained in $R(r, \nu)$, which has dimension

$$
\left(\begin{array}{c}
\nu+2 \\
2
\end{array}\right)-1-\left(\begin{array}{c}
\nu+2-r \\
2
\end{array}\right)
$$

Since a linear space of a given dimension has the maximum possible dimensional space of linear subspaces, we certainly have

$$
\delta(\mu, \nu, r) \leq(\mu+1)\left(\left(\begin{array}{c}
\nu+2 \\
2
\end{array}\right)-1-\left(\begin{array}{c}
\nu+2-r \\
2
\end{array}\right)-\mu\right) .
$$

Even this crude bound has some applications. By Proposition 2.3, we conclude that a general complete intersection $X$ of codimension $m$ in $\mathbb{P}^{n}$ does not have an $r$-Eckardt point if

$$
m\left(\begin{array}{c}
n-m+1-r \\
2
\end{array}\right)>n-m \text {. }
$$

In particular, if $2 m>n$, then at every point of a general complete intersection, there is a quadric of maximal rank in the second fundamental form. Moreover, if

$$
r<n-m-\sqrt{\frac{2(n-m)}{m}},
$$

then a general complete intersection does not have an $r$-Eckardt point. Hence, for giving an upper bound for $\delta(\mu, \nu, r)$, we can assume

$$
r \geq \nu+1-\sqrt{\frac{2(\nu+1)}{\mu+1}} .
$$

Note that if $\mu>1$ or if $\mu=1$ and $\nu>3$, Inequality (3) implies that $3 r>2 \nu$.

We now improve our upper bound for $\delta(\mu, \nu, r)$, subject to $(3)$, using the following simple observation. Let $Q_{1}$ and $Q_{2}$ be two general quadrics in $\Lambda$. Let $V_{1}$ and $V_{2}$ be the singular loci of $Q_{1}$ and $Q_{2}$, respectively. The pencil $\lambda_{1} Q_{1}+\lambda_{2} Q_{2}$ spanned by $Q_{1}$ and $Q_{2}$ has base locus $Q_{1} \cap Q_{2}$. By Bertini's theorem [Ha, III.10.9], the singular locus of a general member of the pencil is contained in the base locus of the pencil. Hence, $V_{1} \cup V_{2} \subset Q_{1} \cap Q_{2}$. Since $Q_{i}$ is a cone with vertex $V_{i}$, we conclude that $\operatorname{Span}\left(V_{1}, V_{2}\right) \subset Q_{1} \cap Q_{2}$.

Pick a general basis $Q_{0}, \ldots, Q_{\mu}$ for $\Lambda$. Let $V_{i}$ denote the singular locus of $Q_{i}$. By the previous observation, to ensure that $\Lambda \subset R(r, \nu)$, we need that each $Q_{i}$ contains $V_{j}$ for all $j$. Notice that this is a necessary condition, but it is not sufficient. Assume that the singular loci of any of the two quadrics intersect in $\mathbb{P}^{a}$ and any three intersect in $\mathbb{P}^{b}$ (since we chose the basis generally, we can assume that these numbers are constant). We also assume that the quadrics have rank $r$. We will now give an upper bound on the dimension of such bases.

Since $Q_{0}$ can be an arbitrary point of $R(r, \nu)$, its choice contributes

$$
\left(\begin{array}{c}
\nu+2 \\
2
\end{array}\right)-1-\left(\begin{array}{c}
\nu+2-r \\
2
\end{array}\right)
$$


to the dimension. Since $V_{1}$ intersects $V_{0}$ in a $\mathbb{P}^{a}$ and $Q_{1}$ contains $V_{0}$ the choice of $Q_{1}$ contributes

$$
(r+a+1)(\nu-r-a)+\left(\begin{array}{c}
r+1 \\
2
\end{array}\right)-\left(\begin{array}{c}
\nu-r-a+1 \\
2
\end{array}\right)-1 .
$$

Next, we choose $Q_{2}$. The vertex $V_{2}$ intersects $V_{0}$ and $V_{1}$ in $a$-dimensional linear spaces and all three intersect in a $b$-dimensional linear space. The family of such $V_{2}$ has dimension

$$
(b+1)(a-b)+2(a-b)(\nu-r-a)+(\nu-r-2 a+b) r .
$$

We then have to choose $Q_{2}$ so that it contains $V_{0}$ and $V_{1}$. This adds

$$
\left(\begin{array}{c}
r+1 \\
2
\end{array}\right)-2\left(\begin{array}{c}
\nu-r-a+1 \\
2
\end{array}\right)+\left(\begin{array}{c}
a-b \\
2
\end{array}\right)-1
$$

to the dimension. The reader can easily verify that under the assumption that $3 r>2 \nu$, both of the choices for $Q_{1}$ and $Q_{2}$ are maximized when $a=b=-1$. We can continue choosing the quadrics in this manner; however, the combinatorics and the issue of whether the linear spaces impose independent conditions get more and more complicated. Since we are not after sharp bounds, we will sidestep all these issues and ignore the additional conditions that are necessary. The dimension for the choice of each of $Q_{3}, \ldots, Q_{\mu}$ is bounded above by the dimension for the choice of $Q_{2}$. Hence, the dimension of the space of such bases is bounded above by

$$
\left(\begin{array}{c}
\nu+2 \\
2
\end{array}\right)+\mu\left((\nu-r+1) r+\left(\begin{array}{c}
r+1 \\
2
\end{array}\right)-1\right)-2 \mu\left(\begin{array}{c}
\nu-r+2 \\
2
\end{array}\right)-1 .
$$

Finally, we can forget the choice of basis of the linear system to conclude the following bounds.

Theorem 2.5. Assume that $\mu \leq\left(\begin{array}{c}r+1 \\ 2\end{array}\right)$ and $2 \nu<3 r$. Then

$$
\begin{array}{r}
(\mu+1)\left(\left(\begin{array}{c}
r+1 \\
2
\end{array}\right)-\mu-1\right)+r(\nu-r+1) \leq \delta(\mu, \nu, r) \\
\leq\left(\begin{array}{c}
\nu+2 \\
2
\end{array}\right)+\mu\left((\nu-r+1) r+\left(\begin{array}{c}
r+1 \\
2
\end{array}\right)-1\right)-2 \mu\left(\begin{array}{c}
\nu-r+2 \\
2
\end{array}\right)-(\mu+1)^{2} .
\end{array}
$$

Combining this with Proposition 2.3, we have the following sufficient conditions for nonexistence of $r$-Eckardt points on a general complete intersection.

Corollary 2.6. Assume $m \geq 3$ or $m=2$ and $n>6$. Let $X$ be a general complete intersection of codimension $m$ in $\mathbb{P}^{n}$.

(1) If

$$
r<\frac{1}{2}+n-m-\frac{1}{3} \sqrt{6\left(\frac{n-m}{m-1}\right)-\frac{15}{4}},
$$

then $X$ does not have any $r$-Eckardt points.

(2) In particular, if $4(m-1)>n-m$, then at every point of $X$ there is a quadric of maximal rank in the second fundamental form. 
Question 2.7. Generalizing from the case of quadrics, one can ask the following question, which would have implications for the geometry of higher fundamental forms. Let $H(k, d, n)$ denote the locus in the Hilbert scheme of hypersurfaces of degree $d$ in $\mathbb{P}^{n}$ parameterizing hypersurfaces whose singular locus has dimension at least $k$. What is the dimension of the space of linear spaces of dimension $m$ in $H(k, d, n)$ ?

Just as for quadrics, by considering the linear system of hypersurfaces that have a fixed $k$-dimensional linear space as singular locus, we can give a lower bound on the dimension of $H(k, d, n)$. Let

$$
M(k, d, n):=\left(\begin{array}{c}
n+d \\
n
\end{array}\right)-\left(\begin{array}{c}
k+d \\
k
\end{array}\right)-(n-k)\left(\begin{array}{c}
k+d-1 \\
k
\end{array}\right)-1 .
$$

If $m \leq M(k, d, n)$, then the dimension of the space of $m$-dimensional linear systems in $H(k, d, n)$ is at least

$$
(m+1)(M(k, d, n)-m)+(k+1)(n-k) .
$$

\section{ECKARDT LOCI ON GENERAL HYPERSURFACES}

In this section, we calculate the class of the $r$-Eckardt locus on a general hypersurface of degree $d$ in $\mathbb{P}^{n}$.

Theorem 3.1. Let $X$ be a general hypersurface of degree $d \geq 3$ in $\mathbb{P}^{n}$. Let $q \geq 0$ be an integer such that $n-1 \geq\left(\begin{array}{c}q+1 \\ 2\end{array}\right)$. Then the $(n-1-q)$-Eckardt locus $X_{n-1-q}$ has codimension $\left(\begin{array}{c}q+1 \\ 2\end{array}\right)$ and degree

$$
\left(\prod_{i=0}^{q-1} \frac{\left(\begin{array}{c}
n+1+i \\
q-i
\end{array}\right)}{\left(\begin{array}{c}
2 i+1 \\
i
\end{array}\right)}\right) d(d-2)^{\frac{q(q+1)}{2}}
$$

The following table lists the degrees of the first five Eckardt loci on a general hypersurface $X$ of degree $d$ in $\mathbb{P}^{n}$.

\begin{tabular}{c|c}
$r$ & degree of $X_{r}$ \\
$n-2$ & $(n+1) d(d-2)$ \\
$n-3$ & $\left(\begin{array}{c}n+2 \\
3\end{array}\right) d(d-2)^{3}$ \\
$n-4$ & $\frac{1}{10}\left(\begin{array}{c}n+3 \\
3\end{array}\right)\left(\begin{array}{c}n+1 \\
3\end{array}\right) d(d-2)^{6}$ \\
$n-5$ & $\frac{1}{21}\left(\begin{array}{c}n+4 \\
5\end{array}\right)\left(\begin{array}{c}n+2 \\
5\end{array}\right) d(d-2)^{10}$ \\
$n-6$ & $\frac{1}{2646}\left(\begin{array}{c}n+5 \\
5\end{array}\right)\left(\begin{array}{c}n+3 \\
5\end{array}\right)\left(\begin{array}{c}n+1 \\
5\end{array}\right) d(d-2)^{15}$
\end{tabular}

Example 3.2 (Flex lines to a plane curve). If $C$ is a plane curve of degree $d$, then the tangent line $L=T_{p} C$ at a point $p \in C$ is a flex line if $\operatorname{mult}_{p}(L \cap C)>2$. Hence, $T_{p} C$ is a flex line if and only if $p$ is a 0 -Eckardt point of $C$. Setting $r=0$ and $n=2$ in Theorem 3.1, we obtain the classical formula that a general plane curve of degree $d$ has $3 d(d-2)$ inflection points [GH2]. In particular, when $d=3$, we get that a cubic curve has 9 inflection points corresponding to the three-torsion points of the elliptic curve. 
The key ingredient in the proof of Theorem 3.1 is the symmetric Porteous formula due to Harris-Tu [HT]. Let $E$ be a vector bundle of rank $n$ and let $L$ be a line bundle. A twisted symmetric map is a map of vector bundles $f: E \rightarrow E^{*} \otimes L$ such that $f$ is locally given by symmetric matrices. The Harris-Tu formula computes the class of the degeneracy locus of a general such map.

Notation 3.3. Let $E$ be a vector bundle of rank $n$ with Chern classes $c_{i}$. Let $0 \leq r \leq n$ be an integer. Define $\mathbb{S}(r, n, E)$ to be the following determinant in the Chern classes of $E$ :

$$
\mathbb{S}(r, n, E):=2^{n-r}\left|\begin{array}{cccccc}
c_{n-r} & c_{n-r+1} & c_{n-r+2} & \ldots & c_{2 n-2 r-2} & c_{2 n-2 r-1} \\
c_{n-r-2} & c_{n-r-1} & c_{n-r} & \ldots & c_{2 n-2 r-4} & c_{2 n-2 r-3} \\
\vdots & & \ddots & & \vdots & \vdots \\
0 & 0 & 0 & \ldots & c_{2} & c_{3} \\
0 & 0 & 0 & \ldots & 1 & c_{1}
\end{array}\right|
$$

Theorem 3.4 (Harris-Tu [HT]). Let $X$ be a compact oriented manifold, E a complex vector bundle of rank $n$ on $X$, and $f: E \rightarrow E^{*} \otimes L$ a general twisted symmetric map. Denote by $X_{r}$ the locus where the map $f$ has rank at most $r$. Then the cohomology class of $X_{r}$ is

$$
\left[X_{r}\right]=\mathbb{S}\left(r, n, E^{*} \otimes \sqrt{L}\right)
$$

where $\sqrt{L}$ is the $\mathbb{Q}$-line bundle with $2 c_{1}(\sqrt{L})=c_{1}(L)$.

Remark 3.5. In the Harris-Tu, one computes the Chern classes of the $\mathbb{Q}$-bundle $E^{*} \otimes \sqrt{L}$ formally using the splitting principle. The final formula involves only integer multiples of the Chern classes of $E^{*}$ and $L$.

We can apply Theorem 3.4 to calculate the classes of Eckardt loci on a general hypersurface $X \subset \mathbb{P}^{n}$. For a smooth hypersurface $X$ embedded in projective space $\mathbb{P}^{n}$ with normal bundle $N_{X} \cong \mathcal{O}_{X}(d)$, the second fundamental form is a map of vector bundles $I I: \operatorname{Sym}^{2} T_{X} \rightarrow N_{X}$, or equivalently a twisted symmetric map $T_{X} \rightarrow T_{X}^{*} \otimes N_{X}$. By definition, the Eckardt loci of $X$ are the degeneracy loci of this map. When $n-1 \geq\left(\begin{array}{c}n-r \\ 2\end{array}\right)$, since the expected class of the $r$-Eckardt locus is non-zero, we conclude that $X_{r}$ is non-empty. Hence, by Theorem 1.3 , the $r$-Eckardt loci have the expected dimension. In particular, their cohomology classes are calculated by the formula of Theorem 3.4 .

Corollary 3.6. Let $X$ be a general hypersurface of degree $d$ in $\mathbb{P}^{n}$. Then the class of the $(n-1-q)$-Eckardt locus is given by the following formula

$$
\left[X_{n-1-q}\right]=\mathbb{S}\left(n-1-q, n-1, T_{X}^{*} \otimes \mathcal{O}_{X}\left(\frac{d}{2}\right)\right)
$$

Proof of Theorem 3.1. In order to prove the theorem, we need to calculate the class in Equation (4). We begin by calculating the Chern classes of $T_{X}^{*} \otimes \mathcal{O}_{X}$. The conormal sequence

$$
0 \rightarrow N_{X}^{*} \rightarrow T_{\left.\mathbb{P}^{n}\right|_{X}}^{*} \rightarrow T_{X}^{*} \rightarrow 0
$$

implies that the $i$ th Chern class of $T_{X}^{*}$ is

$$
c_{i}\left(T_{X}^{*}\right)=\left(\sum_{j=0}^{i}(-1)^{i+j} d^{j}\left(\begin{array}{c}
n+1 \\
i-j
\end{array}\right)\right) h_{X}^{i},
$$


where $h_{X}$ denotes the hyperplane class restricted to $X$. Using the splitting principle, we get the following formula for the Chern classes of $T_{X}^{*} \otimes \mathcal{O}_{X}(d / 2)$ :

$$
\begin{aligned}
c_{i}\left(T_{X}^{*} \otimes \mathcal{O}_{X}\left(\frac{d}{2}\right)\right) & =\sum_{j=0}^{i}\left(\begin{array}{c}
n-1-i+j \\
j
\end{array}\right) c_{i-j}\left(T_{X}^{*}\right)\left(\frac{d}{2}\right)^{j} h_{X}^{j} \\
& =\left(\sum_{m=0}^{i}(-1)^{i+m}\left(\sum_{j=0}^{m}\left(\begin{array}{c}
n-1-i+j \\
j
\end{array}\right) \frac{1}{2^{j}}\right)\left(\begin{array}{c}
n+1 \\
i-m
\end{array}\right) d^{m}\right) h_{X}^{i} .
\end{aligned}
$$

We thus obtain the Chern classes that are the inputs in Equation (4). We need the following lemma in order to evaluate the determinant in Equation (4).

Lemma 3.7. Let $X$ be a smooth hypersurface of degree $d$ in $\mathbb{P}^{n}$. Let $c_{i}$ denote the ith Chern class of the $\mathbb{Q}$-bundle $T_{X}^{*} \otimes \mathcal{O}_{X}(d / 2)$. Then the following formula holds:

$$
c_{i}-\frac{d^{2}}{4} h_{X}^{2} c_{i-2}=\left(\begin{array}{c}
n+1 \\
i
\end{array}\right)\left(\frac{d}{2}-1\right)^{i} h_{X}^{i}
$$

Proof. For the proof of Equation (7), we need the following binomial identity.

$$
A_{m}=\sum_{j=0}^{m} 2^{m-j}\left(\begin{array}{c}
n-1-i+j \\
j
\end{array}\right)-\sum_{j=0}^{m-2} 2^{m-2-j}\left(\begin{array}{c}
n+1-i+j \\
j
\end{array}\right)=\frac{\prod_{j=1}^{m}(n-i+1+j)}{m !}
$$

Equation (8) follows by induction and the recursion

$$
A_{m+1}=2 A_{m}+\left(\begin{array}{c}
n-i+m \\
m+1
\end{array}\right)-\left(\begin{array}{c}
n-i+m \\
m-1
\end{array}\right) .
$$

Using the expression (5) for $c_{i}$, we find that the coefficient of $d^{m}$ on the left hand side of Equation (7) is

$$
(-1)^{i+m}\left(\begin{array}{c}
n+1 \\
i-m
\end{array}\right)\left(\sum_{j=0}^{m}\left(\begin{array}{c}
n-1-i+j \\
j
\end{array}\right) \frac{1}{2^{j}}-\sum_{j=0}^{m-2}\left(\begin{array}{c}
n+1-i+j \\
j
\end{array}\right) \frac{1}{2^{j+2}}\right) .
$$

Factoring out $\frac{1}{2^{m}}$ and using Equation (8), this expression is equal to

$$
(-1)^{i+m}\left(\begin{array}{c}
n+1 \\
i-m
\end{array}\right) \frac{1}{2^{m}} \frac{\prod_{j=1}^{m}(n-i+1+j)}{m !}=(-1)^{i+m} \frac{1}{2^{m}} \frac{(n+1) !}{(n-i+1) ! i !} \frac{i !}{(i-m) ! m !} .
$$

The last expression is equal to

$$
(-1)^{i+m}\left(\begin{array}{c}
n+1 \\
i
\end{array}\right)\left(\begin{array}{c}
i \\
m
\end{array}\right) \frac{1}{2^{m}},
$$

which is precisely the coefficient of $d^{m}$ in

$$
\left(\begin{array}{c}
n+1 \\
i
\end{array}\right)\left(\frac{d}{2}-1\right)^{i}
$$


To conclude the proof of Theorem 3.1, we perform row and column operations on the matrix $\mathbb{S}=$ $\mathbb{S}\left(n-1-q, n-1, T_{X}^{*} \otimes \mathcal{O}_{X}(d / 2)\right)$ to compute the determinant. Let $\rho_{i}(M)$ denote the $i$ th row of a matrix $M$. By Lemma 3.7, replacing $\rho_{i}(\mathbb{S})$ with $\rho_{i}(\mathbb{S})-\frac{d^{2}}{4} h_{X}^{2} \rho_{i+1}(\mathbb{S})$ for $i=1, \ldots, q-1$ in order yields

$$
\mathbb{S}=2^{q}\left|\begin{array}{cccc}
\left(\begin{array}{c}
n+1 \\
q
\end{array}\right)\left(\frac{d}{2}-1\right)^{q} & \left(\begin{array}{c}
n+1 \\
q+1
\end{array}\right)\left(\frac{d}{2}-1\right)^{q+1} & \ldots & \left(\begin{array}{c}
n+1 \\
2 q-1
\end{array}\right)\left(\frac{d}{2}-1\right)^{2 q-1} \\
\left(\begin{array}{c}
n+1 \\
q-2
\end{array}\right)\left(\frac{d}{2}-1\right)^{q-2} & \left(\begin{array}{c}
n+1 \\
q-1
\end{array}\right)\left(\frac{d}{2}-1\right)^{q-1} & \ldots & \left(\begin{array}{c}
n+1 \\
2 q-3
\end{array}\right)\left(\frac{d}{2}-1\right)^{2 q-3} \\
\vdots & 0 & \ddots & \\
0 & \ldots & (n+1)\left(\frac{d}{2}-1\right)
\end{array}\right| h_{X}^{\frac{q(q+1)}{2}}
$$

If we expand this matrix using permutations, every term has a $\left(\frac{d}{2}-1\right)^{\frac{q(q+1)}{2}}$ factor. Hence, we conclude that

$$
\mathbb{S}=2^{q}\left(\frac{d}{2}-1\right)^{\frac{q(q+1)}{2}} h_{X}^{\frac{q(q+1)}{2}}\left|\begin{array}{cccc}
\left(\begin{array}{c}
n+1 \\
q
\end{array}\right) & \left(\begin{array}{c}
n+1 \\
q+1
\end{array}\right) & \ldots & \left(\begin{array}{c}
n+1 \\
2 q-1
\end{array}\right) \\
\left(\begin{array}{c}
n+1 \\
q-2
\end{array}\right) & \left(\begin{array}{c}
n+1 \\
q-1
\end{array}\right) & \ldots & \left(\begin{array}{c}
n+1 \\
2 q-3
\end{array}\right) \\
\vdots & & \ddots & \\
0 & 0 & \ldots & (n+1)
\end{array}\right| .
$$

The latter determinant is well-known to be

$$
\left|\begin{array}{cccc}
\left(\begin{array}{c}
n+1 \\
q
\end{array}\right) & \left(\begin{array}{c}
n+1 \\
q+1
\end{array}\right) & \ldots & \left(\begin{array}{c}
n+1 \\
2 q-1
\end{array}\right) \\
\left(\begin{array}{c}
n+1 \\
q-2
\end{array}\right) & \left(\begin{array}{c}
n+1 \\
q-1
\end{array}\right) & \ldots & \left(\begin{array}{c}
n+1 \\
2 q-3
\end{array}\right) \\
\vdots & & \ddots & \\
0 & 0 & \ldots & (n+1)
\end{array}\right|=2^{\left(\begin{array}{c}
q \\
2
\end{array}\right)} \prod_{i=0}^{q-1} \frac{\left(\begin{array}{c}
n+1+i \\
q-i
\end{array}\right)}{\left(\begin{array}{c}
2 i+1 \\
i
\end{array}\right)}
$$

and can be easily computed by reducing the matrix to an upper triangular matrix via a series of column operations. We refer the reader to $[\mathrm{ACGH}]$ or $[\mathrm{HT}]$ for the proof. We conclude that

$$
\mathbb{S}=\left(\prod_{i=0}^{q-1} \frac{\left(\begin{array}{c}
n+1+i \\
q-i
\end{array}\right)}{\left(\begin{array}{c}
2 i+1 \\
i
\end{array}\right)}\right)(d-2)^{\frac{q(q+1)}{2}} h_{X}^{\frac{q(q+1)}{2}}
$$

Since the degree of $h_{X}^{\frac{q(q+1)}{2}}$ is $d$, Theorem 3.1 follows.

\section{ECKARDT LOCI IN A GENERAL PENCIL OF HYPERSURFACES}

Throughout this section, let $n=\left(\begin{array}{c}q+1 \\ 2\end{array}\right)$. By Theorem 1.3, the locus of hypersurfaces that have an $(n-1-q)$-Eckardt point is a divisor in the Hilbert scheme of hypersurfaces of degree $d$. The main theorem in this section computes the degree of this divisor. The main new feature is the need to extend Theorem 3.4 from vector bundles to coherent sheaves that fail to be locally free at singular points of the fibers of the pencil.

Let $\pi: X \rightarrow \mathbb{P}^{1}$ be a general pencil of hypersurfaces of degree $d$ in $\mathbb{P}^{n}$. For our generality assumption, we require that

(1) the total space $X$ of the pencil is smooth, and 
(2) the pencil intersects the discriminant transversely at points corresponding to hypersurfaces with a unique $A_{1}$ singularity.

With these assumptions, we have the following theorem.

Theorem 4.1. Let $n=\left(\begin{array}{c}q+1 \\ 2\end{array}\right)$. Let $\pi: X \rightarrow \mathbb{P}^{1}$ be a general pencil of hypersurfaces of degree $d \geq 3$ in $\mathbb{P}^{n}$. Then the number of hypersurfaces in the pencil that have an $(n-1-q)$-Eckardt point is

$$
\left(\prod_{i=0}^{q-1} \frac{\left(\begin{array}{c}
n+1+i \\
q-i
\end{array}\right)}{\left(\begin{array}{c}
2 i+1 \\
i
\end{array}\right)}\right)(d-2)^{n-1}((n+1) d-2) .
$$

The following table lists these numbers for the first five values of $q$.

\begin{tabular}{|c|c|c|}
\hline$q$ & $n$ & \# hypersurfaces with an $(n-1-q)$-Eckardt point in a pencil in $\mathbb{P}^{n}$ \\
\hline 2 & 3 & $10(4 d-2)(d-2)^{2}$ \\
\hline 3 & 6 & $294(7 d-2)(d-2)^{5}$ \\
\hline 4 & 10 & $75504(11 d-2)(d-2)^{9}$ \\
\hline 5 & 15 & $219,288,576(16 d-2)(d-2)^{14}$ \\
\hline 6 & 21 & $8,809,061,000,025(22 d-2)(d-2)^{20}$ \\
\hline
\end{tabular}

Remark 4.2. The number of surfaces in a general pencil in $\mathbb{P}^{3}$ having a 0-Eckardt point was also calculated by Rosenberg [Ros] using the relative jet bundle of the family. More generally, Rosenberg's technique can compute the number of 0-Eckardt points in a general $\left(\begin{array}{c}n-1 \\ 2\end{array}\right)$-dimensional linear system of hypersurfaces.

Proof of Theorem 4.1. The cohomology ring of $\mathbb{P}^{1} \times \mathbb{P}^{n}$ is

$$
H^{*}\left(\mathbb{P}^{1} \times \mathbb{P}^{n}, \mathbb{Z}\right) \cong \frac{\mathbb{Z}[x, y]}{\left\langle x^{2}, y^{n+1}\right\rangle},
$$

where $x$ and $y$ are the pullbacks of the hyperplane classes on $\mathbb{P}^{1}$ and $\mathbb{P}^{n}$, respectively. The total space of the pencil $X$ is a divisor of bi-degree $(1, d)$ in $\mathbb{P}^{1} \times \mathbb{P}^{n}$; therefore, the normal bundle $N_{X}$ of $X$ in the product $\mathbb{P}^{1} \times \mathbb{P}^{n}$ has first Chern class

$$
c_{1}\left(N_{X}\right)=x+d y
$$

where, by an abuse of notation, we denote the restrictions of the classes $x$ and $y$ to $X$ by the same symbols. It is easy to calculate the intersection numbers $x y^{n-1}=d$ and $y^{n}=1$.

Now consider the exact sequence of coherent sheaves on $X$

$$
0 \rightarrow \pi^{*} \Omega_{\mathbb{P}^{1}} \rightarrow \Omega_{X} \rightarrow \Omega_{\pi} \rightarrow 0,
$$

where the first map is given by pullback of 1-forms. By Whitney's formula, the Chern classes of $\Omega_{\pi}$ are given by

$$
c_{i}\left(\Omega_{\pi}\right)=(-1)^{i+1} \sum_{j=0}^{i}\left(\begin{array}{c}
n+1 \\
j
\end{array}\right)\left(d^{i-j-1} x y^{i-1}+d^{i-j} y^{i}\right) .
$$


Although an exact sequence of coherent sheaves, the sequence $(9)$ is not an exact sequence of vector bundles. If the morphism $\pi$ is smooth at a point $p \in X$ contained in the fiber $X_{t}$, then $\left(\Omega_{\pi}\right)_{p}=\left(\Omega_{X_{t}}\right)_{p}$. However, at singular points of the fibers of $\pi$, the map $\Omega_{\mathbb{P}^{1}} \rightarrow \Omega_{X}$ becomes zero, so the cokernel $\Omega_{\pi}$ fails to be locally free.

The formula in Theorem 3.4 a priori applies only to vector bundles. In order to obtain a symmetric Porteous formula in our setting, we first resolve the map in the sequence (9) (see Diaz [D] for a general strategy for extending Porteous-type formulas to morphisms of coherent sheaves). We need to blow up $X$ along the $(n-1)$ st Fitting ideal of $\Omega_{\pi}([\mathrm{D}],[\mathrm{Li}])$. An easy local calculation shows that the $(n-1)$ st Fitting ideal of $\Omega_{\pi}$ is the maximal ideal at each of the singular points of the fibers of the map $\pi$. Briefly, at a singular point of the map $\pi$, the pencil is locally given by the equation $\sum_{i=0}^{n} x_{i}^{2}=t$. Hence, $\Omega_{X}$ is generated by $d x_{0}, \ldots, d x_{n}, d t$ subject to the relation that $\sum_{i=0}^{n} 2 x_{i} d x_{i}=d t$. Therefore, the $(n-1)$ st Fitting ideal of $\Omega_{\pi}$ is generated by the $2 \times 2$ minors of the matrix

$$
\left(\begin{array}{ccccc}
2 x_{0} & 2 x_{1} & \cdots & 2 x_{n} & 1 \\
0 & 0 & \cdots & 0 & 1
\end{array}\right)
$$

We conclude that the Fitting ideal is $\mathcal{I}_{Z}$, where $Z$ is the union of the singular points of the fibers of $\pi$.

Let $p: X^{\prime} \rightarrow X$ be the blowup of $X$ along $Z$, and let $E$ denote the exceptional divisor. The map $\pi^{*} \Omega_{\mathbb{P}^{1}} \rightarrow \Omega_{X}$ is zero precisely along $Z$, so there is an injective map of sheaves $\pi^{*} \Omega_{\mathbb{P}^{1}} \otimes I_{Z} \rightarrow \Omega_{X}$. Pulling back via the blowup $p$, we get an injective map of vector bundles

$$
p^{*} \pi^{*} \Omega_{\mathbb{P}^{1}} \otimes \mathcal{O}(-E) \rightarrow p^{*} \Omega_{X}
$$

The cokernel of this map is then a vector bundle $\widetilde{\Omega}_{\pi}$ which is isomorphic to $\Omega_{\pi}$ under the identification of $X \backslash Z$ and $X^{\prime} \backslash p^{-1}(Z)$. If we define $\widetilde{T}_{\pi}$ to be the dual of this vector bundle, then $\widetilde{T}_{\pi}$ is an extension of (the pullback of) $\left(T_{\pi}\right) \mid X \backslash Z$, and by Diaz [D, Theorem 1] the second fundamental form extends to a map of vector bundles $\operatorname{Sym}^{2} \widetilde{T}_{\pi} \rightarrow p^{*} N_{X}$. Since the singular members of the pencil have only $A_{1}$ singularities, a local calculation shows that the map has full rank at every point of the exceptional divisor $E$. Hence, by Theorem 3.4, we can calculate the classes of Eckardt loci of members of the family in terms of the Chern classes of the $\mathbb{Q}$-bundle $\widetilde{\Omega}_{\pi} \otimes \sqrt{p^{*} N_{X}}$.

To calculate these Chern classes, consider the conormal bundle sequence for the inclusion $X \subset \mathbb{P}^{1} \times \mathbb{P}^{n}$ :

$$
0 \rightarrow N_{X}^{*} \rightarrow\left(\Omega_{\mathbb{P}^{1}} \otimes \Omega_{\mathbb{P}^{n}}\right)_{\mid X} \rightarrow \Omega_{X} \rightarrow 0 .
$$

Taking Chern polynomials, we get

$$
c\left(\Omega_{X}\right)=\frac{(1-y)^{n+1}(1-2 x)}{1-x-d y} .
$$

Next, on $X^{\prime}$ we have the exact sequence of vector bundles

$$
0 \rightarrow p^{*} \pi^{*} \Omega\left(\mathbb{P}^{1}\right) \otimes \mathcal{O}(-E) \rightarrow p^{*} \Omega_{X} \rightarrow \widetilde{\Omega}_{\pi} \rightarrow 0
$$

so we get

$$
c\left(\widetilde{\Omega_{\pi}}\right)=\frac{(1-y)^{n+1}(1-2 x)}{(1-x-d y)(1-2 x-e)},
$$

where by slight abuse of notation we use $x$ and $y$ to denote the pullback to $X^{\prime}$ of the corresponding classes on $X$, and $e$ denotes the class of the exceptional divisor of $p$. 
Using the fact that $e \cdot x=e \cdot y=0$ in the cohomology of the blowup, we can rewrite

$$
c\left(\widetilde{\Omega_{\pi}}\right)=\frac{(1-y)^{n+1}}{(1-x-d y)}+e \sum_{i=0}^{n-1} e^{i}=p^{*} c\left(\Omega_{\pi}\right)+e \sum_{i=0}^{n-1} e^{i} .
$$

Using the splitting principle, we see that the Chern classes of $\widetilde{\Omega_{\pi}} \otimes \sqrt{p^{*} N_{X}}$ are given by

$$
c_{i}\left(\widetilde{\Omega_{\pi}} \otimes \sqrt{p^{*} N_{X}}\right)=\sum_{j=0}^{i}\left(\begin{array}{c}
n-1-j \\
i-j
\end{array}\right)\left(p^{*} c_{j}\left(\Omega_{\pi}\right)+e^{j}\right)\left(\frac{x}{2}+\frac{d}{2} y\right)^{i-j}
$$

Expanding the right hand side using the cohomology relations $x^{2}=x \cdot e=y \cdot e=0$, we obtain

$$
e^{i}+\sum_{j=0}^{i}\left(\begin{array}{c}
n-1-j \\
i-j
\end{array}\right) \frac{1}{2^{i-j}}\left(\sum_{l=0}^{j}(-1)^{l}\left(\begin{array}{c}
n+1 \\
l
\end{array}\right)\left((i-l) d^{i-l-1} x y^{i-1}+d^{i-l} y^{i}\right)\right) .
$$

First, we deal with the case when $q=2$ and $n=3$. Using the cohomology relations $x^{2}=y^{4}=0$, $e^{i} \cdot x^{j}=e^{i} \cdot y^{j}=0$ for $j>0$, from 12 we obtain the following Chern classes for the twisted rank-2 bundle $\widetilde{\Omega_{\pi}} \otimes \sqrt{p^{*} N_{X}}$ :

$$
\begin{aligned}
& \widetilde{c}_{1}=2 x+(2 d-4) y+e \\
& \widetilde{c}_{2}=\left(\frac{7 d}{2}-6\right) x y+\left(\frac{7 d^{2}}{4}-6 d+6\right) y^{2}+e^{2} .
\end{aligned}
$$

By Theorem 3.4, the locus of Eckardt points of surfaces in the pencil has class $4 \widetilde{c}_{1} \widetilde{c}_{2}$. The pencil has $4(d-1)^{3}$ singular fibers. Since the exceptional divisor has one component of self-intersection -1 for each of these fibers, we conclude that $e^{3}=-4 \cdot(d-1)^{3}$. Using the intersection numbers $x y^{2}=d$ and $y^{3}=1$, we obtain that the number of 0 -Eckardt points in the pencil is $10(4 d-2)(d-2)^{2}$ as claimed.

Now assume that $q>2$. In this case, we can simplify the determinant

$$
\left|\begin{array}{cccc}
c_{q}\left(\Omega_{\pi}\right)+e^{q} & c_{q+1}\left(\Omega_{\pi}\right)+e^{q+1} & \ldots & c_{2 q-1}\left(\Omega_{\pi}\right)+e^{2 q-1} \\
\vdots & & \ddots & \\
0 & 0 & \ldots & c_{1}\left(\Omega_{\pi}\right)+e
\end{array}\right|
$$

by column operations. If $q$ is even, multiply the first column by $e$ and subtract it from the second column. If $q$ is odd, multiply the second column by $e$ and subtract it from the third column. In each case, we obtain a determinant with one column with positive degree entries that do not have any terms with $e$. Consequently, by the multilinearity of the determinant, we conclude that this determinant is equal to

$$
\mathbb{S}\left(n-1-q, n-1, \Omega_{\pi} \otimes \mathcal{O}_{X}\left(\frac{1}{2} x+\frac{d}{2} y\right)\right) .
$$

It remains to calculate this determinant. We begin with a recurrence relation for the Chern classes similar to Lemma 3.7. 
Lemma 4.3. The Chern classes $c_{i}\left(\Omega_{\pi} \otimes \mathcal{O}_{X}\left(\frac{1}{2} x+\frac{d}{2} y\right)\right)$ satisfy the relation

$$
\begin{aligned}
& c_{i}-\left(x y+\frac{d^{2}}{4} y^{2}\right) c_{i-2}-\sum_{m=1}^{\left\lfloor\frac{i}{2}\right\rfloor-1}(d-1)\left(\frac{d}{2}-1\right)^{2 m-1} c_{i-2-2 m} \\
& =\left(\frac{d}{2}-1\right)^{i-1} y^{i-1}\left(A_{i} x+B_{i}(d-2) y\right)
\end{aligned}
$$

where

$$
A_{i}=\frac{1}{2}\left(\sum_{j=0}^{i} \frac{i}{2^{i-j}}\left(\begin{array}{c}
n-1-j \\
i-j
\end{array}\right)-\frac{1}{4} \sum_{j=0}^{i-2} \frac{i-2}{2^{i-j}}\left(\begin{array}{c}
n-1-j \\
i-2-j
\end{array}\right)\right) \quad \text { and } \quad B_{i}=\frac{1}{2}\left(\begin{array}{c}
n+1 \\
i
\end{array}\right) .
$$

Proof. The proof of the stated formula for $B_{i}$ is exactly as in Lemma 3.7.

We were unable to prove the stated formula for $A_{i}$ by hand. Instead, one can use algorithms of WilfZeilberger type to verify this identity. In more detail, using formula (12) one can calculate the coefficient of $x y^{i}$ appearing in the first line of the statement of Lemma 4.3. The resulting expression can then be input into the Mathematica package HolonomicFunctions developed by Koutschan [K], allowing to prove algorithmically the desired identity. We are grateful to Christoph Koutschan who carried out this calculation for us.

Using Lemma 4.3 , we can evaluate the determinant $\mathbb{S}$ as follows. Let

$$
\alpha_{i}=\left(A_{i}-i B_{i}\right) x y^{i-1} \text { and } \beta_{i}=B_{i}\left(x+\left(\frac{d}{2}-1\right) y\right)^{i} .
$$

The lemma shows that, by row operations, we can replace $c_{i}$ by $\left.\left(\frac{d}{2}-1\right)^{i-1} y^{i-1}\left(A_{i} x+B_{i}(d-2) y\right)\right)$. This, in turn, is equal to $\beta_{i}+\alpha_{i}$ since $x^{2}=0$. Expanding using multilinearity, we get

$$
\mathbb{S}=\left|\begin{array}{ccc}
\beta_{q} & \beta_{q+1} & \cdots \\
\beta_{q-2} & \beta_{q-1} & \cdots \\
\vdots & \ddots & \vdots \\
0 & \cdots & \beta_{1}
\end{array}\right|+\left|\begin{array}{ccc}
\alpha_{q} & \alpha_{q+1} & \cdots \\
\beta_{q-2} & \beta_{q-1} & \cdots \\
\vdots & \ddots & \vdots \\
0 & \cdots & \beta_{1}
\end{array}\right|+\left|\begin{array}{ccc}
\beta_{q} & \beta_{q+1} & \cdots \\
\alpha_{q-2} & \alpha_{q-1} & \cdots \\
\vdots & \ddots & \vdots \\
0 & \cdots & \beta_{1}
\end{array}\right|+\cdots+\left|\begin{array}{ccc}
\beta_{q} & \beta_{q+1} & \cdots \\
\beta_{q-2} & \beta_{q-1} & \cdots \\
\vdots & \ddots & \vdots \\
0 & \cdots & \alpha_{1}
\end{array}\right|,
$$

where each determinant has at most one row consisting of $\alpha_{i}$ 's since $x^{2}=0$ in the cohomology ring.

Observe that all the determinants in (13) except for the first one are zero. This is because the row consisting of $\alpha_{i}$ 's is dependent on the rows below it as we will now show. Let $D_{i}=\sum_{j=0}^{i}\left(\begin{array}{c}n-1-i+j \\ j\end{array}\right)$. Then the expressions for $B_{i}, A_{i}$ and Lemma 4.3 yield the two relations

$$
A_{i}-i B_{i}=2\left(D_{i}-B_{i}\right) \text { and } B_{i}=D_{i}-\frac{1}{4} D_{i-2}
$$

Hence, we recursively obtain the linear relation

$$
\left(A_{i}-i B_{i}\right)-2 \sum_{\substack{j=1 \\ 15}}^{\left\lfloor\frac{i}{2}\right\rfloor} \frac{1}{4^{j}} B_{i-2 j}=0 .
$$


We conclude that there is the following linear relation

$$
\alpha_{i}-\sum_{j=1}^{\left\lfloor\frac{i}{2}\right\rfloor} \frac{x y^{2 j-1}}{4^{j}}(d-2)^{2 j} \beta_{i-2 j}=0
$$

among the rows of all the matrices in 13 except for the first one. It follows that all the determinants except for the first one are zero.

Finally,

$$
\mathbb{S}=\left|\begin{array}{ccc}
\beta_{q} & \beta_{q+1} & \cdots \\
\beta_{q-2} & \beta_{q-1} & \cdots \\
\vdots & \ddots & \vdots \\
0 & \cdots & \beta_{1}
\end{array}\right|=(x+(d-2) y)^{n}\left(\frac{1}{2}\right)^{n}\left|\begin{array}{ccc}
B_{i} & B_{i+1} & \cdots \\
B_{i-1} & B_{i-1} & \cdots \\
\vdots & \ddots & \vdots \\
0 & \cdots & B_{1}
\end{array}\right|
$$

Using the intersection numbers $x y^{n-1}=d$ and $y^{n}=1$, we see that $(x+(d-2) y)^{n}=(d-2)^{n-1}((n+1) d-2)$. Finally, the last determinant was computed in the proof of Theorem 3.1. Putting these together gives the claimed formula for $\mathbb{S}$.

\section{REFERENCES}

[ACGH] E. Arbarello, M. Cornalba, P. Griffiths and J. Harris, Geometry of Algebraic Curves, Grundlehren der mathematischen Wissenschaften, Springer, 1985.

[C] I. Coskun, Restriction varities and geometric branching rules, Adv. Math., 228 no. 4 (2011), 2441-2502.

[CS] I. Coskun and J. Starr, Rational curves on smooth cubic hypersurfaces, Int. Math. Res. Not., RPN 102 (2009), $4626-4641$.

[D] S. Diaz, Porteous' formula for maps between coherent sheaves. Michigan Math. J., 52 (2004), no. 3, $507-514$.

[GH] P. Griffiths and J. Harris, Algebraic geometry and local differential geometry, Ann. Sci. Éc. Norm. Sup., 12 no. 3 (1979), $355-452$.

[GH2] P. Griffiths and J. Harris, Principles of algebraic geometry, Wiley Interscience, 1978.

[HT] J. Harris and L. Tu, On symmetric and skew-symmetric determinantal varieties, Topology 23 no.1 (1984), 71-84.

[Ha] R. Hartshorne, Algebraic Geometry, Springer 1978.

[IL] B. Ilic and J.M. Landsberg, On symmetric degeneracy loci, spaces of symmetric matrices of constant rank and dual varieties, Math. Ann. 314 (1999), 159-174.

[K] C. Koutschan, Advanced applications of the holonomic systems approach, Ph.D. Thesis, Research Institute for Symbolic Computation (RISC), Johannes Kepler University, 2009.

[L] J.M. Landsberg, On the second fundamental form of projective varieties, Invent. Math., 117 no. 2 (1994), 303-316.

[LR] J.M. Landsberg and C. Robles. Fubini's theorem in codimension two. J. Reine. Angew. Math. 631 (2009), $221-235$.

[Li] J. Lipman, On the Jacobian ideal of the module of differentials, Proc. Amer. Math. Soc., 21 (1969), $422-426$.

[M] R. Meshulam, On two extremal matrix problems, Linear Algebra Appl., 114/115 (1989), 261-271.

[Ros] J. Rosenberg, Geometry of moduli of cubic surfaces, Ph.D. Thesis, University of Michigan, 1999.

[W1] R. Westwick, Spaces of matrices of fixed rank, Linear and Multilinear Algebra, 20 (1987), 171-174.

[W2] R. Westwick, Spaces of matrices of fixed rank II, Linear Algebra Appl., 235 (1996), 163-169.

University of Illinois at Chicago, Department of Mathematics, Statistics and Computer Science, Chicago, IL 60607

E-mail address: coskun@math.uic.edu, artie@math.uic.edu 